Supporting information

\title{
Electrocatalytic Formal [2+2] Cycloaddition Reactions between Anodically Activated Aliphatic Enol Ethers and Nonactivated Olefins Possessing an Alkoxyphenyl Group
}

\author{
Yohei Okada ${ }^{1}$, Ryoichi Akaba ${ }^{2}, K^{2}$ azuhiro Chiba*1 \\ ${ }^{1}$ Laboratory of Bio-organic Chemistry, Tokyo University of Agriculture and Technology, \\ 3-5-8 Saiwai-cho, Fuchu, 183-8509 Tokyo, Japan \\ Tel:+81-42-367-5667, Fax:+81-42-360-7167 \\ chiba@cc.tuat.ac.jp \\ ${ }^{2}$ Department of Chemistry, Gunma College of Technology, 580 Toriba-machi, Maebashi, \\ Gunma 371-8530, Japan
}

\section{General Information}

${ }^{1} \mathrm{H}$ and ${ }^{13} \mathrm{C}$ NMR spectra were recorded in $\mathrm{CDCl}_{3}$ with TMS as an initial standard on JEOL AL600 (600 MHz and $150 \mathrm{MHz}$ respectively) spectrometer. The following abbreviations were used to explain multiplicities: s, singlet; d, doublet; t, triplet; $q$, quartet; quint, quintet; sext, sextet; sept, septet; m, multiple. TLC analysis was carried out with Merk Silica Gel $60 \mathrm{~F}_{254}$ plates, detection of compounds was achieved by UV absorption $(254 \mathrm{~nm})$ and by charring after spraying with 12 molybdo(VI) phosphoric acid $n$-hydrate in $95 \%$ ethanol. Column chromatography was performed with Kieselgel 60 Silica Gel (Merk, 0.04- $0.063 \mathrm{~mm}$ ). All reagents and solvents were purchased from Kanto chemical, Tokyo Kasei Kogyo, Aldrich and Wako and used as received. Mass spectra were measured using JEOL JMS-K9 and JMS-700. Redox potentials were measured by cyclic voltammetry (Hokuto Denko, HSV-100) by using glassy carbon (working electrode), $\mathrm{Pt}$ wire (counter electrode) and $\mathrm{Ag} / \mathrm{AgCl}$ (reference electrode).

\section{Experimental Information}

Olefins possessing an alkoxyphenyl group $(4.0 \mathrm{mmol})$ and aliphatic enol ethers $(0.20$ mmol) were added to $1.0 \mathrm{M} \mathrm{LiClO}{ }_{4} \mathrm{CH}_{3} \mathrm{NO}_{2}(20 \mathrm{~mL})$. The reaction cell was capped with a septum equipped with the carbon felt anode carbon felt cathode $(20 \mathrm{~mm} \times 20$ $\mathrm{mm}$ ), and the $\mathrm{Ag} / \mathrm{AgCl}$. The electrolysis was then performed at $1.2 \mathrm{~V}$ (vs. $\mathrm{Ag} / \mathrm{AgCl}$ ). After the completion of the reaction (ca. 0.3-0.5 F/mol), the reaction mixture was 
poured into EtOAc, and the EtOAc solution was successively washed with brine. The organic layer was dried over anhydrous $\mathrm{MgSO}_{4}$. After filtration and evaporation under reduced pressure, the residue was purified by silica gel column chromatography using $n$-hexane-EtOAc to give cycloadducts. The products yields were determined by GC-MS.

\section{Spectral Information}

\section{1-(2-Ethoxy-3-methyl-cyclobutylmethyl)-4-methoxy-benzene 1a}

${ }^{1} \mathrm{H} \mathrm{NMR}\left(\mathrm{CDCl}_{3}, 600 \mathrm{MHz}\right) \delta 7.07(2 \mathrm{H}, \mathrm{d}, J=8.1 \mathrm{~Hz}), 6.81(2 \mathrm{H}, \mathrm{d}, J=8.1 \mathrm{~Hz}), 3.78(3 \mathrm{H}$, s), $3.43(2 \mathrm{H}, \mathrm{m}), 3.16(1 \mathrm{H}, \mathrm{t}, J=7.3 \mathrm{~Hz}), 2.88(1 \mathrm{H}, \mathrm{dd}, J=13.9,5.1 \mathrm{~Hz}) 2.55(1 \mathrm{H}, \mathrm{dd}$, $J=13.9,9.5 \mathrm{~Hz}), 2.22(1 \mathrm{H}, \mathrm{m}), 2.02(1 \mathrm{H}, \mathrm{sept}, J=7.3 \mathrm{~Hz}) 1.94(1 \mathrm{H}, \mathrm{dd}, J=14.7,8.7 \mathrm{~Hz})$, $1.17(3 \mathrm{H}, \mathrm{t}, J=7.3 \mathrm{~Hz}), 1.10(3 \mathrm{H}, \mathrm{d}, J=6.6 \mathrm{~Hz}), 0.77(1 \mathrm{H}, \mathrm{dd}, J=14.7,10.3 \mathrm{~Hz}){ }^{13} \mathrm{C} \mathrm{NMR}$ $\left(\mathrm{CDCl}_{3}, 150 \mathrm{MHz}\right) \delta 157.7,132.8,129.5,113.6,85.5,64.1,55.2,42.1,40.1,35.7,27.0$, 19.8, 15.5 IR ( $\left.\mathrm{NaCl}, \mathrm{cm}^{-1}\right)$ 2952, 2930, 2867, 1512, 1245, 1177, 1119, 1038 MS (rel. int.) $\mathrm{m} / \mathrm{z}$ 234( $\left.\mathrm{M}^{+}, 5\right), 192(66), 148(100), 121(90), 91(48)$, 77(54), HRMS calc. for $\mathrm{C}_{15} \mathrm{H}_{22} \mathrm{O}_{2} 234.1620$, found 234.1630

\section{1-(2-Ethoxy-3-methyl-cyclobutylmethyl)-4-methoxy-benzene 1b-c}

${ }^{1} \mathrm{H} \mathrm{NMR}\left(\mathrm{CDCl}_{3}, 600 \mathrm{MHz}\right) \delta 7.07(2 \mathrm{H}, \mathrm{d}, J=8.8 \mathrm{~Hz}), 6.81(2 \mathrm{H}, \mathrm{d}, J=8.8 \mathrm{~Hz}), 3.78(3 \mathrm{H}$, s), $3.55(1 \mathrm{H}, \mathrm{t}, J=7.3 \mathrm{~Hz}), 3.39$ (2H, m), $2.90(1 \mathrm{H}, \mathrm{dd}, J=13.2,4.4 \mathrm{~Hz}), 2.61(1 \mathrm{H}, \mathrm{m})$, $2.55(1 \mathrm{H}, \mathrm{dd}, J=13.2,9.5 \mathrm{~Hz}), 1.39(1 \mathrm{H}, \mathrm{m}), 1.26(1 \mathrm{H}, \mathrm{m}), 1.18(1 \mathrm{H}, \mathrm{m}), 1.17(3 \mathrm{H}, \mathrm{t}$, $J=7.3), 1.11(3 \mathrm{H}, \mathrm{d}, J=6.6 \mathrm{~Hz}){ }^{13} \mathrm{C} \mathrm{NMR}\left(\mathrm{CDCl}_{3}, 150 \mathrm{MHz}\right) \delta 157.7,132.8,129.6$, 113.6, 78.8, 64.3, 55.2, 42.7, 39.6, 31.4, 26.8, 15.3, 14.2 IR $\left(\mathrm{NaCl}, \mathrm{cm}^{-1}\right) 2960,2929$, 2868, 1512, 1246, 1176, 1132, $1038 \mathrm{MS}$ (rel. int.) m/z 234( $\left.\mathrm{M}^{+}, 2\right), 192(52), 148(100)$, 121(36), 91(22), 77(26), HRMS calc. for $\mathrm{C}_{15} \mathrm{H}_{22} \mathrm{O}_{2}\left(\mathrm{M}^{+}\right)$234.1620, found 234.1594

${ }^{1} \mathrm{H}$ NMR $\left(\mathrm{CDCl}_{3}, 600 \mathrm{MHz}\right) \delta 7.10(2 \mathrm{H}, \mathrm{d}, J=8.8 \mathrm{~Hz}), 6.81(2 \mathrm{H}, \mathrm{d}, J=8.8 \mathrm{~Hz}), 3.78(3 \mathrm{H}$, s), $3.58(1 \mathrm{H}, \mathrm{t}, J=7.3 \mathrm{~Hz}), 3.39(2 \mathrm{H}, \mathrm{m}), 2.88(1 \mathrm{H}, \mathrm{dd}, J=13.2,4.4 \mathrm{~Hz}), 2.61(1 \mathrm{H}, \mathrm{m})$, $2.45(1 \mathrm{H}, \mathrm{m}), 1.65(1 \mathrm{H}, \mathrm{m}), 1.40(1 \mathrm{H}, \mathrm{m}), 1.19(3 \mathrm{H}, \mathrm{t}, J=7.3 \mathrm{~Hz}), 1.17(1 \mathrm{H}, \mathrm{m}), 1.05$ $(3 \mathrm{H}, \mathrm{d}, J=7.3 \mathrm{~Hz}){ }^{13} \mathrm{C} \mathrm{NMR}\left(\mathrm{CDCl}_{3}, 150 \mathrm{MHz}\right) \delta 157.6,133.6,129.8,113.6,81.0,64.5$, $55.2,38.8,36.3,33.6,29.7,26.2,19.4$

\section{8-(4-Methoxy-benzyl)-2-oxa-bicyclo[4.2.0]octane 2a}

${ }^{1} \mathrm{H} \mathrm{NMR}\left(\mathrm{CDCl}_{3}, 600 \mathrm{MHz}\right) \delta 7.08(2 \mathrm{H}, \mathrm{d}, J=8.8 \mathrm{~Hz}), 6.82(2 \mathrm{H}, \mathrm{d}, J=8.8 \mathrm{~Hz}), 3.87(1 \mathrm{H}$, t, $J=6.6 \mathrm{~Hz}), 3.78(3 \mathrm{H}, \mathrm{s}), 3.73-3.65(1 \mathrm{H}, \mathrm{m}), 3.63-3.56(1 \mathrm{H}, \mathrm{m}), 2.88-2.78(2 \mathrm{H}, \mathrm{m})$, $2.58(1 \mathrm{H}, \mathrm{dd}, J=15.4,10.3 \mathrm{~Hz}), 2.40-2.30(1 \mathrm{H}, \mathrm{m}), 1.96-1.86(1 \mathrm{H}, \mathrm{m}), 1.72-1.64(1 \mathrm{H}$, 
m), 1.60-1.51 (1H, m), 1.51-1.42 (2H, m), 1.42-1.34 (1H, m) ${ }^{13} \mathrm{C} \mathrm{NMR}\left(\mathrm{CDCl}_{3}, 150\right.$ $\mathrm{MHz}) \delta 157.7,132.9,129.5,113.7,74.7,63.0,55.2,39.4,38.8,30.4,26.8,25.7,23.3$ IR

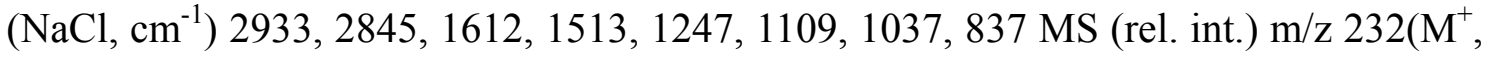
12), 148(83), 133(53), 121(100), 105(35), 84(65), HRMS calc. for $\mathrm{C}_{15} \mathrm{H}_{21} \mathrm{O}_{2}\left(\mathrm{M}^{+}+\mathrm{H}\right)$ 233.1542, found 233.1527

\section{8-(4-Methoxy-benzyl)-2-oxa-bicyclo[4.2.0]octane 2b}

${ }^{1} \mathrm{H} \mathrm{NMR}\left(\mathrm{CDCl}_{3}, 600 \mathrm{MHz}\right) \delta 7.11(2 \mathrm{H}, \mathrm{d}, J=8.8 \mathrm{~Hz}), 6.80(2 \mathrm{H}, \mathrm{d}, J=8.8 \mathrm{~Hz}), 4.11(1 \mathrm{H}$, $\mathrm{dd}, J=8.1,4.4 \mathrm{~Hz}), 3.92-3.87(1 \mathrm{H}, \mathrm{m}), 3.78(3 \mathrm{H}, \mathrm{s}), 3.25-3.18(1 \mathrm{H}, \mathrm{m}), 2.82(1 \mathrm{H}, \mathrm{dd}$, $J=13.9,8.1 \mathrm{~Hz}), 2.54(1 \mathrm{H}, \mathrm{dd}, J=13.9,8.1 \mathrm{~Hz}), 2.34-2.24(1 \mathrm{H}, \mathrm{m}), 2.18-2.09(1 \mathrm{H}, \mathrm{m})$, $1.99(1 \mathrm{H}, \mathrm{dd}, J=20.5,10.3 \mathrm{~Hz}), 1.91-1.84(1 \mathrm{H}, \mathrm{m}), 1.84-1.77(1 \mathrm{H}, \mathrm{m}), 1.60-1.49(2 \mathrm{H}$, $\mathrm{m}), 1.47-1.40(1 \mathrm{H}, \mathrm{m}){ }^{13} \mathrm{C} \mathrm{NMR}\left(\mathrm{CDCl}_{3}, 150 \mathrm{MHz}\right) \delta 157.5,133.8,129.5,113.6,75.0$, 64.7, 55.2, 39.3, 33.7, 30.2, 29.1, 23.4, 21.7 IR $\left(\mathrm{NaCl}, \mathrm{cm}^{-1}\right)$ 2933, 2849, 1612, 1512 , 1245, 1176, 1040, $833 \mathrm{MS}$ (rel. int.) $\mathrm{m} / \mathrm{z}$ 232( $\left.\mathrm{M}^{+}, 12\right)$, 148(90), 133(47), 121(100), 105(32), 84(68), HRMS calc. for $\mathrm{C}_{15} \mathrm{H}_{21} \mathrm{O}_{2} 233.1542\left(\mathrm{M}^{+}+\mathrm{H}\right)$, found 233.1539

\section{7-(4-Methoxy-benzyl)-2-oxa-bicyclo[3.2.0]heptane 3a}

${ }^{1} \mathrm{H} \mathrm{NMR}\left(\mathrm{CDCl}_{3}, 600 \mathrm{MHz}\right) \delta 7.08(2 \mathrm{H}, \mathrm{d}, J=8.8 \mathrm{~Hz}), 6.81(2 \mathrm{H}, \mathrm{d}, J=8.8 \mathrm{~Hz}), 4.31-4.25$ $(1 \mathrm{H}, \mathrm{m}), 4.12-4.05(1 \mathrm{H}, \mathrm{m}), 3.97-3.90(1 \mathrm{H}, \mathrm{m}), 3.77(3 \mathrm{H}, \mathrm{s}), 2.97-2.89(1 \mathrm{H}, \mathrm{m}), 2.80$ $(1 \mathrm{H}, \mathrm{dd}, J=13.9,7.3 \mathrm{~Hz}), 2.63(1 \mathrm{H}, \mathrm{dd}, J=13.9,8.8 \mathrm{~Hz}), 2.42-2.32(1 \mathrm{H}, \mathrm{m}), 1.87-1.77$ $(1 \mathrm{H}, \mathrm{m}), 1.76-1.68(1 \mathrm{H}, \mathrm{m}), 1.68-1.62(1 \mathrm{H}, \mathrm{m}), 1.62-1.54(1 \mathrm{H}, \mathrm{m}){ }^{13} \mathrm{C} \mathrm{NMR}\left(\mathrm{CDCl}_{3}\right.$, $150 \mathrm{MHz}) \delta 157.8,132.4,129.4,113.7,82.9,67.5,55.2,41.0,39.2,35.2,32.1,27.3$ IR $\left(\mathrm{NaCl}, \mathrm{cm}^{-1}\right)$ 2933, 2853, 1612, 1512, 1248, 1065, 1038, $830 \mathrm{MS}$ (rel. int.) m/z 218( $\mathrm{M}^{+}$, 23), 148(77), 133(50), 121(100), 91(52), 70(66), HRMS calc. for $\mathrm{C}_{14} \mathrm{H}_{19} \mathrm{O}_{2}\left(\mathrm{M}^{+}+\mathrm{H}\right)$ 219.1385, found 219.1357

\section{7-(4-Methoxy-benzyl)-2-oxa-bicyclo[3.2.0]heptane 3b}

${ }^{1} \mathrm{H} \mathrm{NMR}\left(\mathrm{CDCl}_{3}, 600 \mathrm{MHz}\right) \delta 7.11(2 \mathrm{H}, \mathrm{d}, J=8.8 \mathrm{~Hz}), 6.80(2 \mathrm{H}, \mathrm{d}, J=8.8 \mathrm{~Hz}), 4.62-4.48$ $(1 \mathrm{H}, \mathrm{m}), 4.20(1 \mathrm{H}, \mathrm{t}, J=8.1 \mathrm{~Hz}), 4.10-4.00(1 \mathrm{H}, \mathrm{m}), 3.77(3 \mathrm{H}, \mathrm{s}), 2.94(1 \mathrm{H}$, quint, $J=6.6$ $\mathrm{Hz}), 2.78(1 \mathrm{H}, \mathrm{dd}, J=13.9,8.1 \mathrm{~Hz}), 2.57(1 \mathrm{H}),, 2.44(1 \mathrm{H}, \mathrm{dd}, J=13.9,8.1 \mathrm{~Hz}), 2.30-2.17$ $(1 \mathrm{H}, \mathrm{m}), 1.86-1.74(1 \mathrm{H}, \mathrm{m}), 1.67(1 \mathrm{H}, \mathrm{dd}, J=12.5,5.9 \mathrm{~Hz}), 1.38-1.23(1 \mathrm{H}, \mathrm{m}){ }^{13} \mathrm{C} \mathrm{NMR}$ $\left(\mathrm{CDCl}_{3}, 150 \mathrm{MHz}\right) \delta 157.6,133.3,129.5,113.6,80.7,69.6,55.2,39.5,36.2,34.2,31.8$, 29.7 IR ( $\left.\mathrm{NaCl}, \mathrm{cm}^{-1}\right)$ 2933, 2857, 1611, 1512, 1245, 1083, 1038, $832 \mathrm{MS}$ (rel. int.) $\mathrm{m} / \mathrm{z}$ 218( $\left.\mathrm{M}^{+}, 29\right), 148(83), 133(50), 121(100), 91(50), 70(72)$, HRMS calc. for $\mathrm{C}_{15} \mathrm{H}_{21} \mathrm{O}_{2}$ $\left(\mathrm{M}^{+}+\mathrm{H}\right) 219.1385$, found 219.1357 


\section{8-(2-Methoxy-benzyl)-2-oxa-bicyclo[4.2.0]octane 4a}

${ }^{1} \mathrm{H} \mathrm{NMR}\left(\mathrm{CDCl}_{3}, 600 \mathrm{MHz}\right) \delta 7.17(1 \mathrm{H}, \mathrm{t}, J=8.1 \mathrm{~Hz}), 7.12(1 \mathrm{H}, \mathrm{d}, J=7.3 \mathrm{~Hz}), 6.88(1 \mathrm{H}$, t, $J=7.3 \mathrm{~Hz}), 6.83(1 \mathrm{H}, \mathrm{d}, J=8.1 \mathrm{~Hz}), 3.88(1 \mathrm{H}, \mathrm{t}, J=6.6 \mathrm{~Hz}), 3.81(3 \mathrm{H}, \mathrm{s}), 3.75-3.67(1 \mathrm{H}$, m), 3.62-3.54 (1H, m), 2.94-2.85 (2H, m), $2.66(1 \mathrm{H}, \mathrm{dd}, J=16.1,10.3 \mathrm{~Hz}), 2.42-2.33$ $(1 \mathrm{H}, \mathrm{m}), 1.95-1.84(1 \mathrm{H}, \mathrm{m}), 1.74-1.65(1 \mathrm{H}, \mathrm{m}), 1.58-1.52(1 \mathrm{H}, \mathrm{m}), 1.52-1.44(2 \mathrm{H}, \mathrm{m})$, 1.44-1.37 (1H, m) ${ }^{13} \mathrm{C} \mathrm{NMR}\left(\mathrm{CDCl}_{3}, 150 \mathrm{MHz}\right) \delta 157.5,129.9,129.2,126.9,120.3$, 110.1, 75.0, 63.1, 55.2, 37.9, 33.6, 30.4, 27.0, 25.7, $23.3 \mathrm{IR}\left(\mathrm{NaCl}, \mathrm{cm}^{-1}\right)$ 2937, 2845, 1600, 1494, 1243, 1113, 1031, $752 \mathrm{MS}$ (rel. int.) m/z 232( $\left.\mathrm{M}^{+}, 5\right), 148(100), 133(47)$, 119(78), 105(42), 91(94), HRMS calc. for $\mathrm{C}_{15} \mathrm{H}_{21} \mathrm{O}_{2}\left(\mathrm{M}^{+}+\mathrm{H}\right)$ 233.1542, found 233.1539

\section{8-(3,4-Dimethoxy-benzyl)-2-oxa-bicyclo[4.2.0]octane 5a}

${ }^{1} \mathrm{H}$ NMR $\left(\mathrm{CDCl}_{3}, 600 \mathrm{MHz}\right) \delta$ 6.86-6.68 (3H, m), 3.96-3.84 (7H, m), 3.78-3.68 (1H, m), 3.68-3.58 (1H, m), 2.95-2.81 (2H, m), $2.60(1 \mathrm{H}, \mathrm{dd}, \mathrm{J}=16.1,10.3 \mathrm{~Hz}), 2.46-2.33(1 \mathrm{H}$, $\mathrm{m}), 2.01-1.88(1 \mathrm{H}, \mathrm{m}), 1.79-1.66(1 \mathrm{H}, \mathrm{m}), 1.58-1.53(1 \mathrm{H}, \mathrm{m}), 1.53-1.50(2 \mathrm{H}, \mathrm{m})$, 1.50-1.37 (1H, m) ${ }^{13} \mathrm{C} \mathrm{NMR}\left(\mathrm{CDCl}_{3}, 150 \mathrm{MHz}\right) \delta 148.7,147.1,133.5,120.3,112.0$, 111.1, 74.8, 63.0, 55.9, 55.8, 39.4, 39.3, 30.4, 26.8, 25.7, $23.3 \mathrm{IR}\left(\mathrm{NaCl}, \mathrm{cm}^{-1}\right) 2933$, 2842, 1590, 1516, 1261, 1238, 1031, $765 \mathrm{MS}$ (rel. int.) m/z 262( $\left.\mathrm{M}^{+}, 28\right), 178(100)$, 163(55), 147(72), 107(44), 91(43), HRMS calc. for $\mathrm{C}_{16} \mathrm{H}_{22} \mathrm{O}_{3}\left(\mathrm{M}^{+}\right) 262.1569$, found 262.1572 


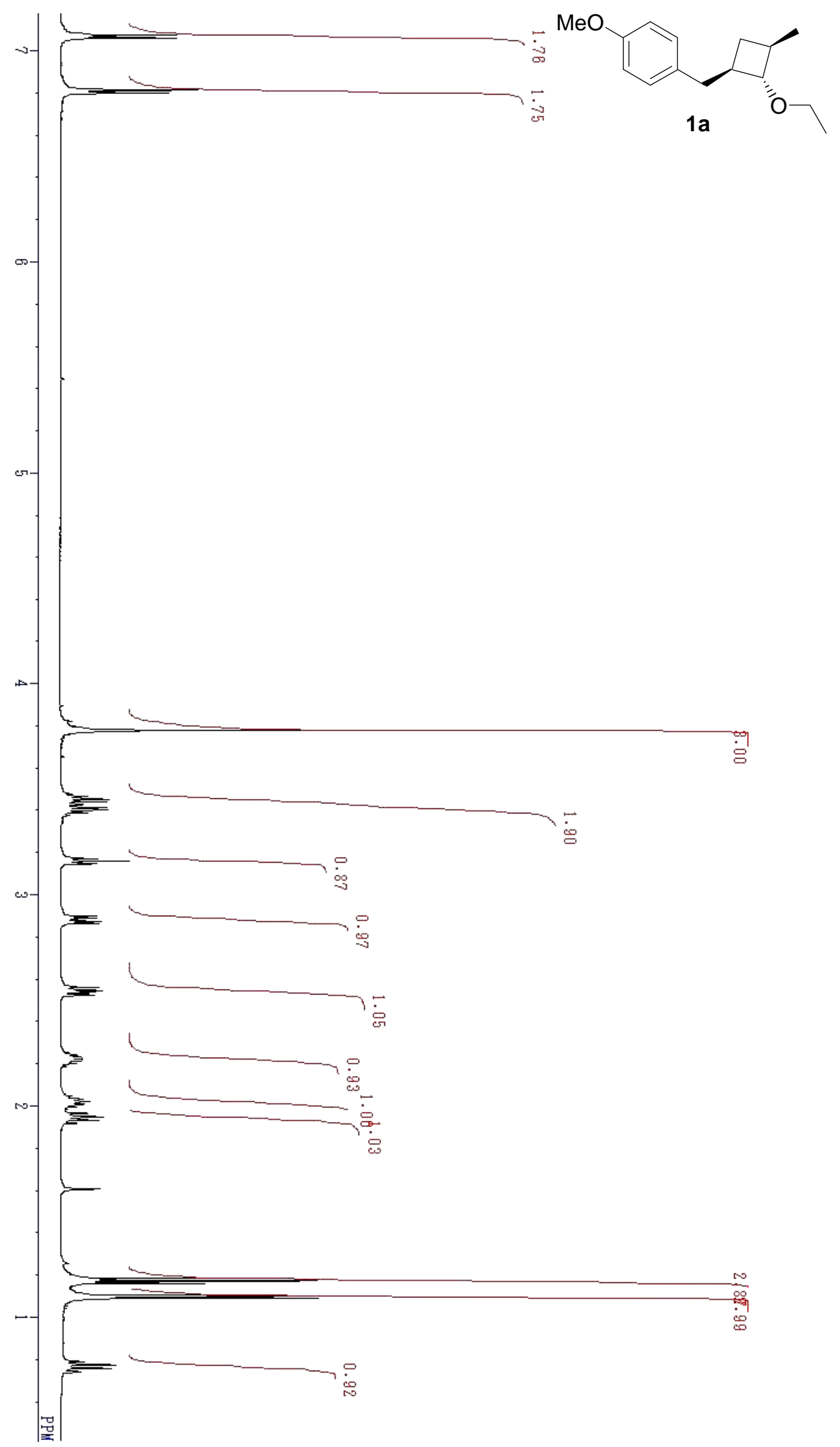




$$
1
$$




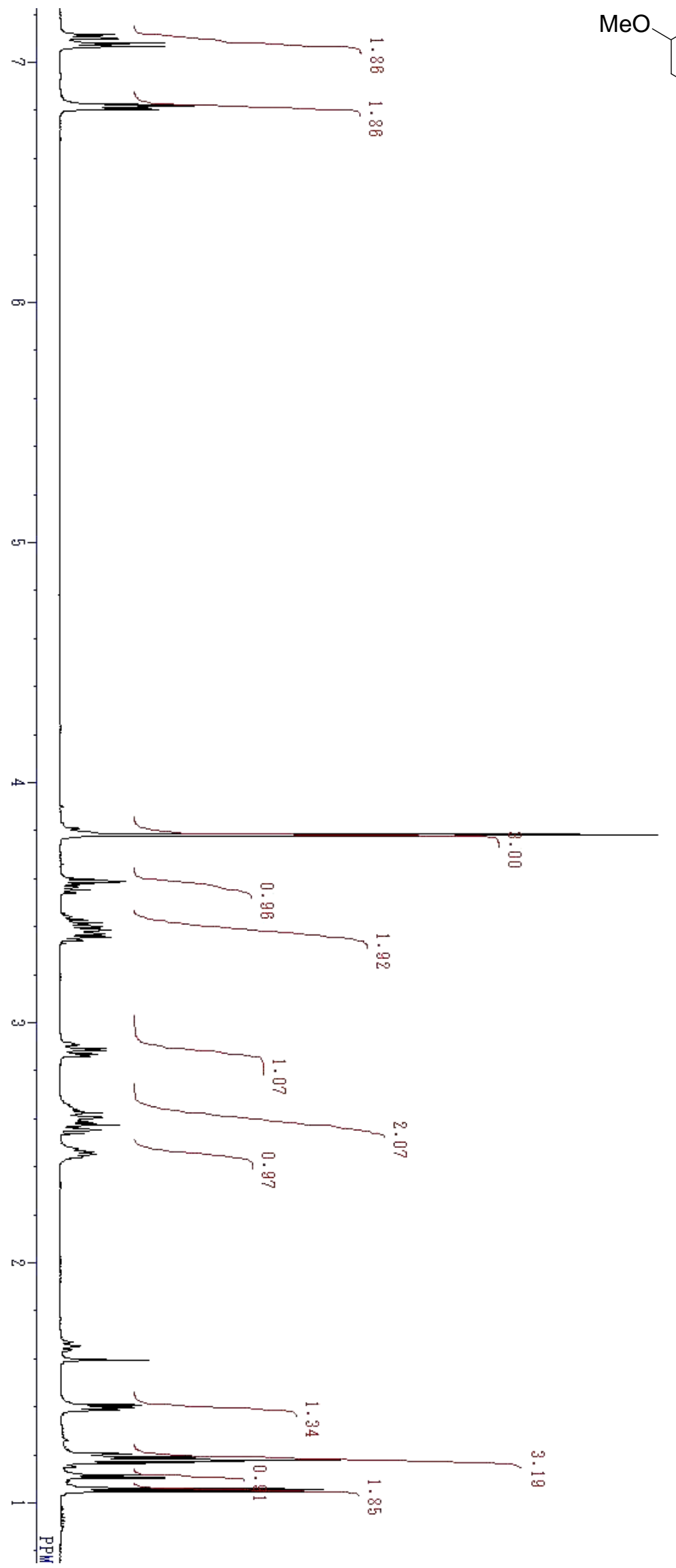




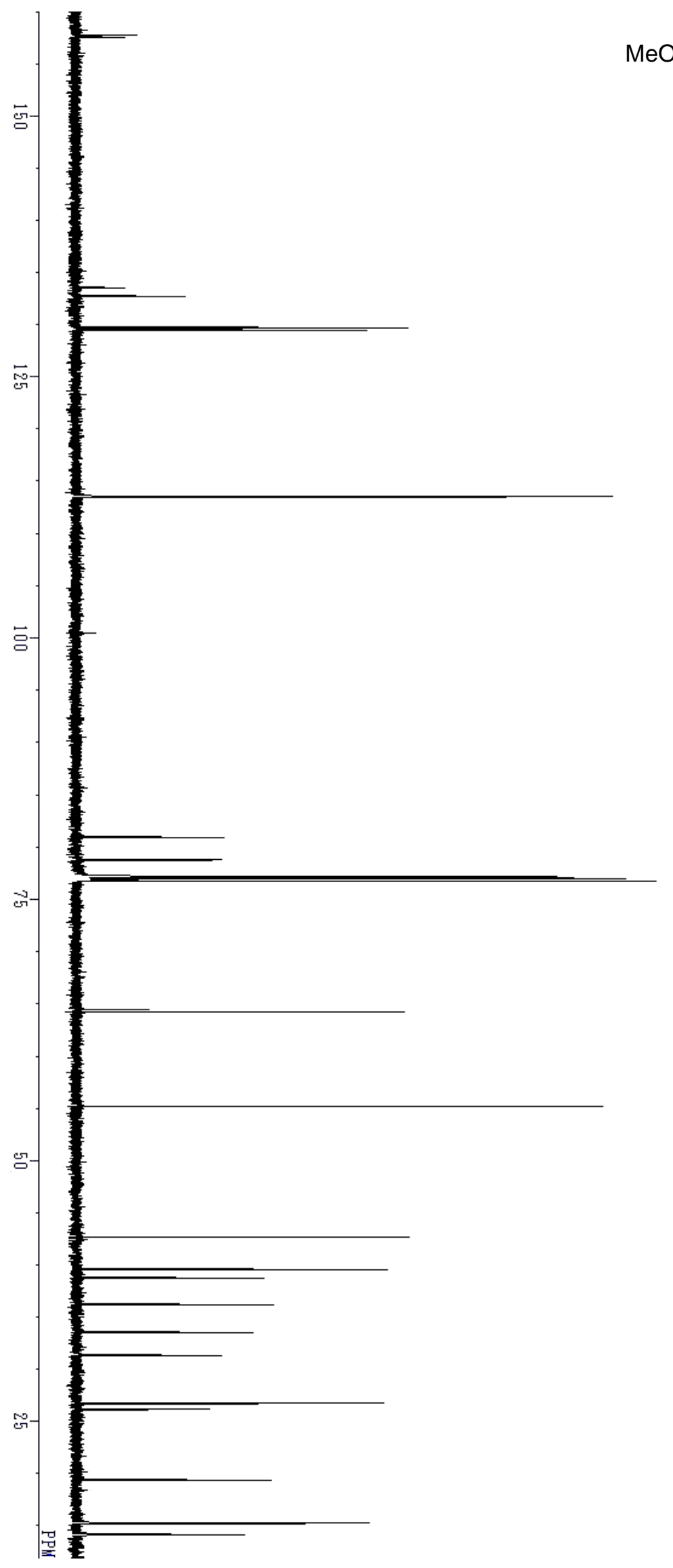




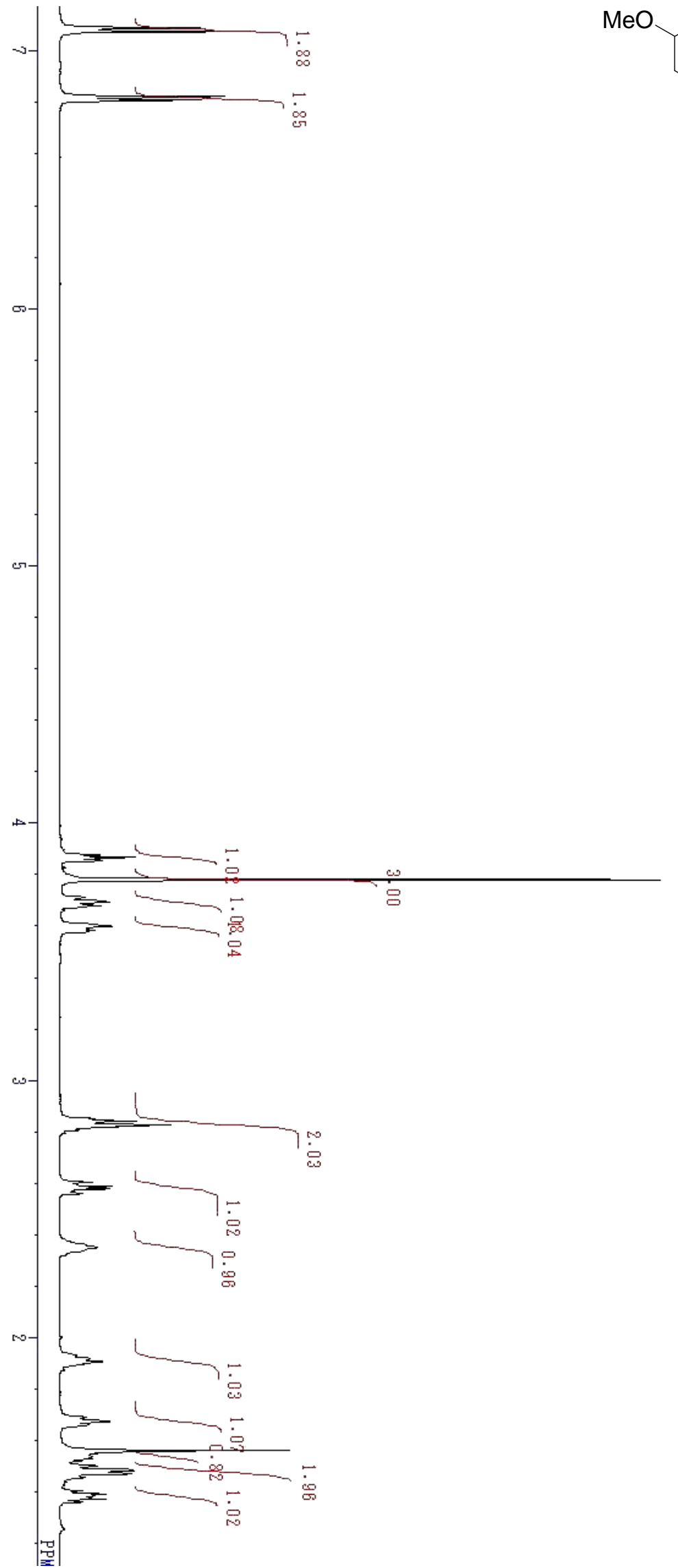

$2 a$ 


$$
E
$$




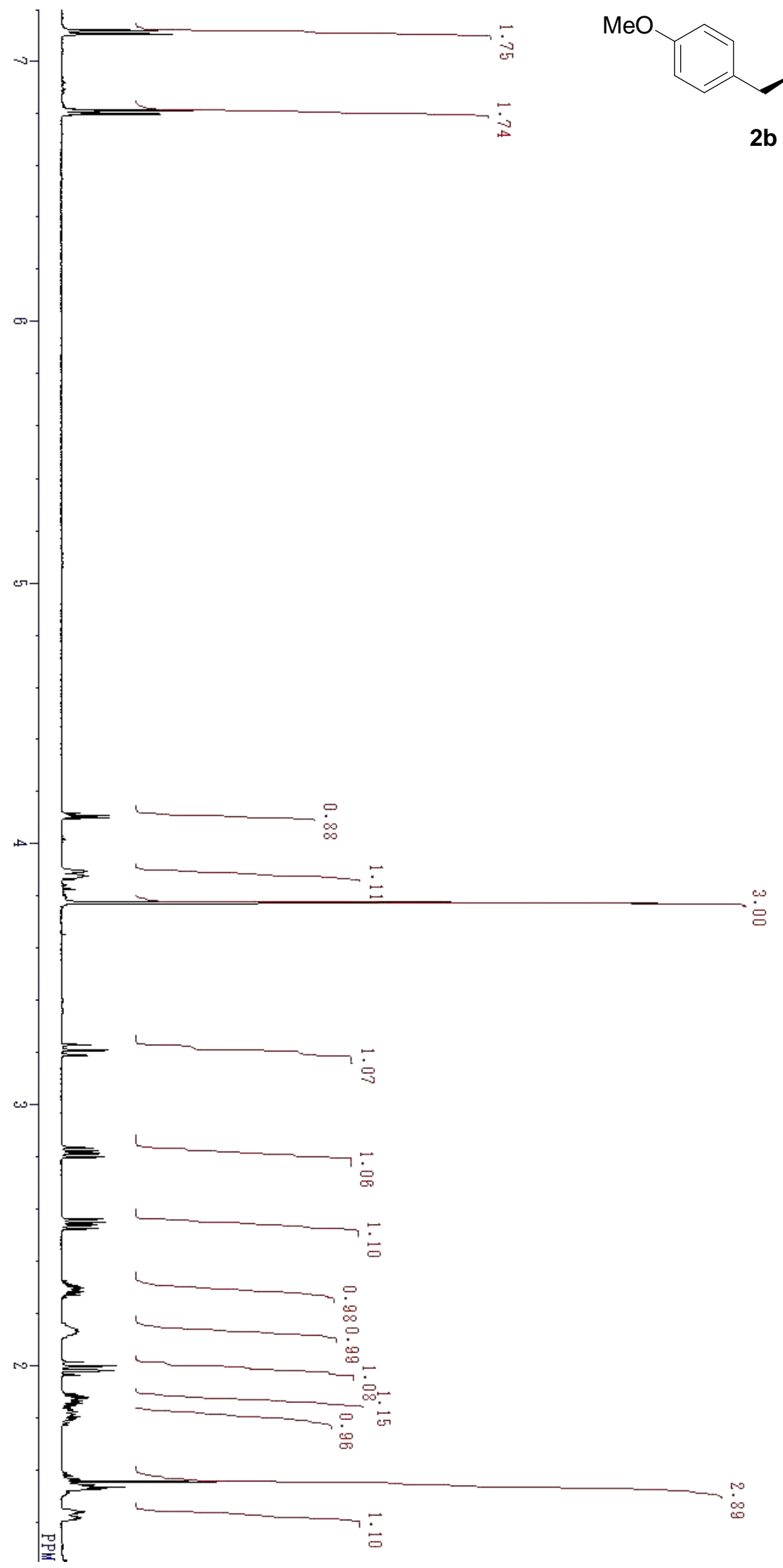




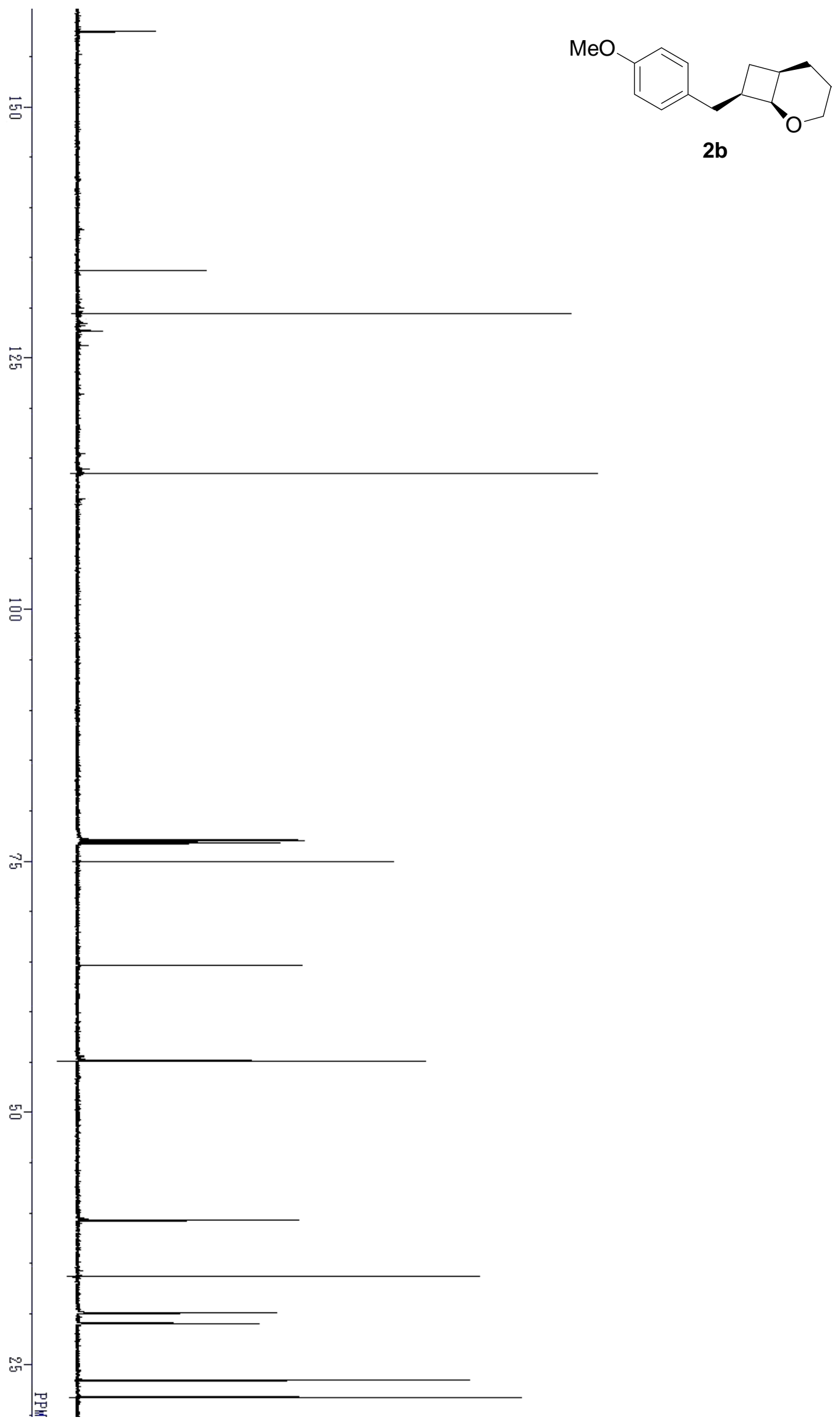




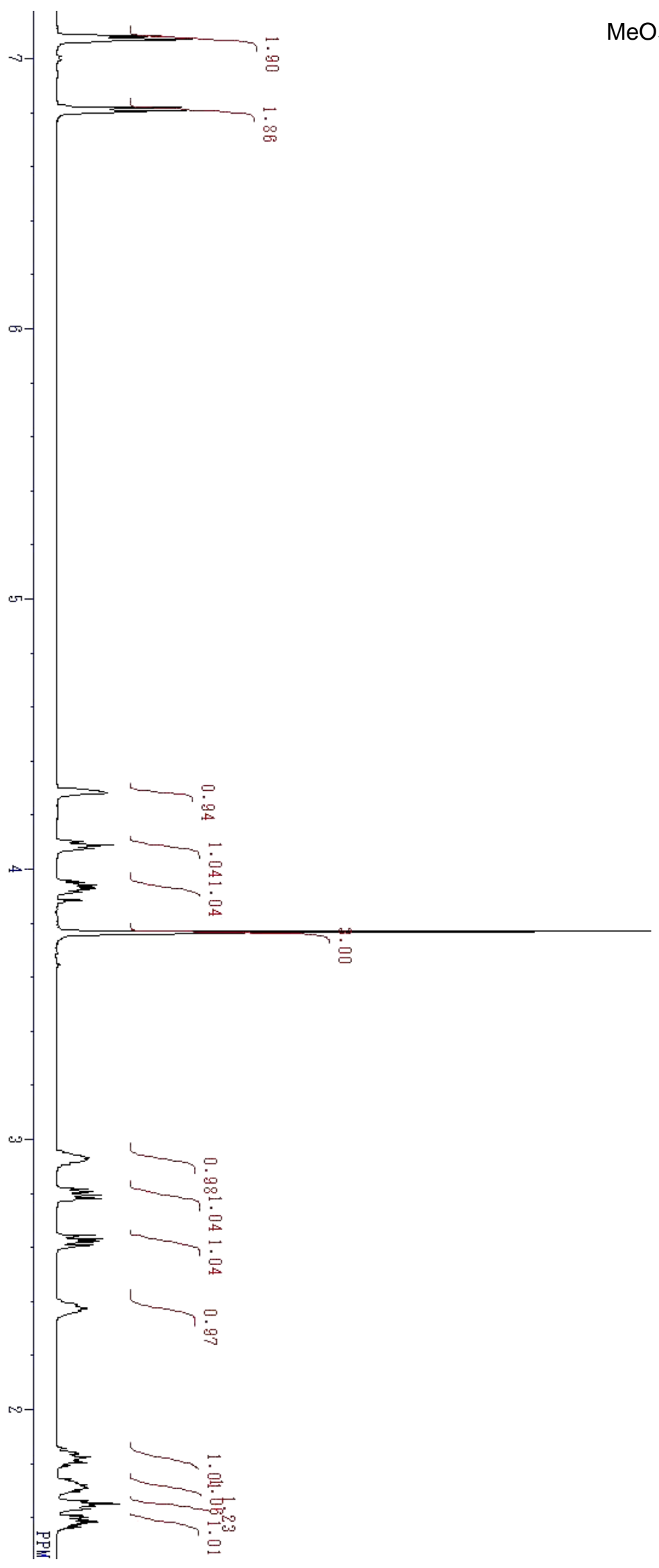

$3 a$ 


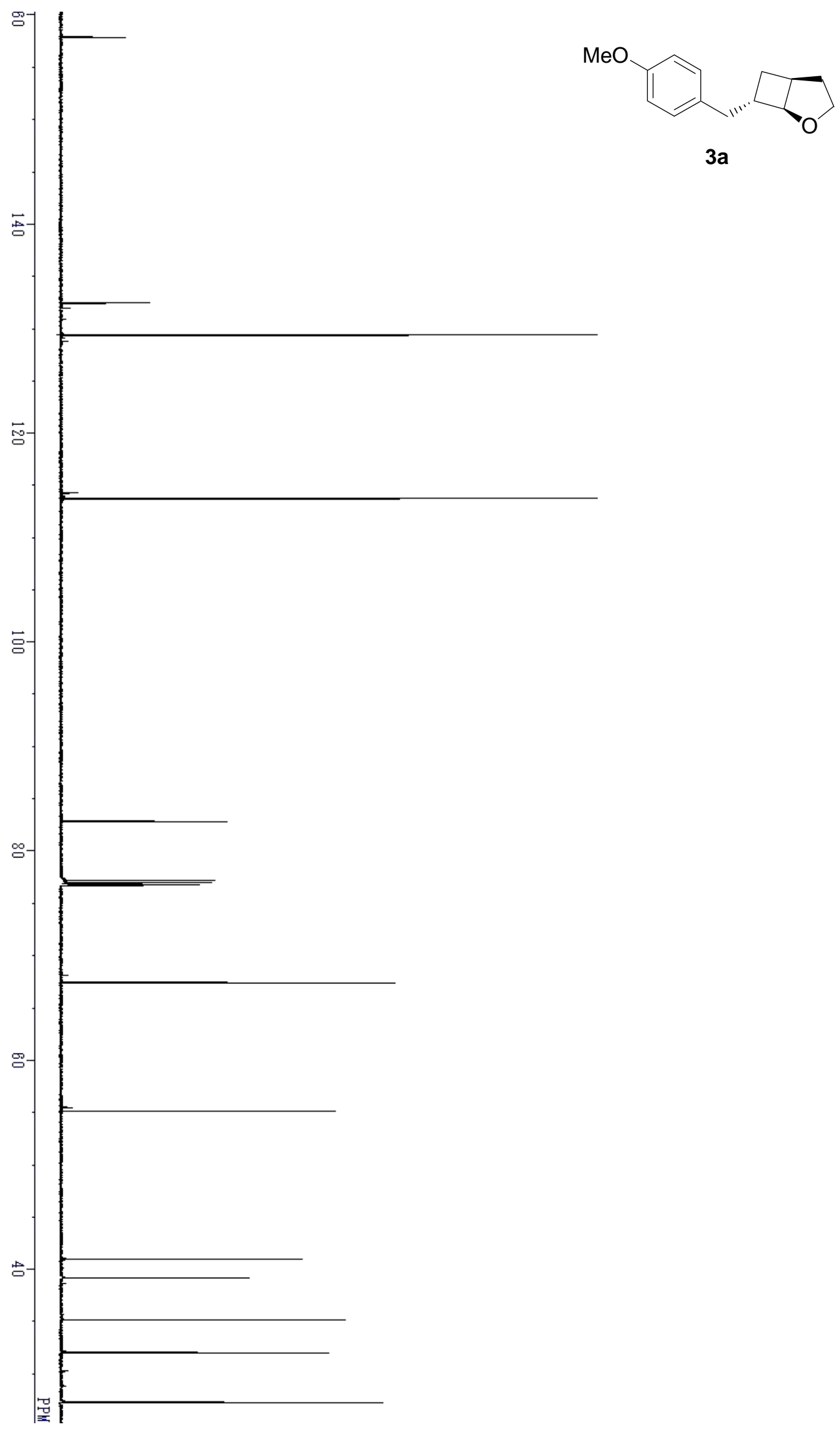




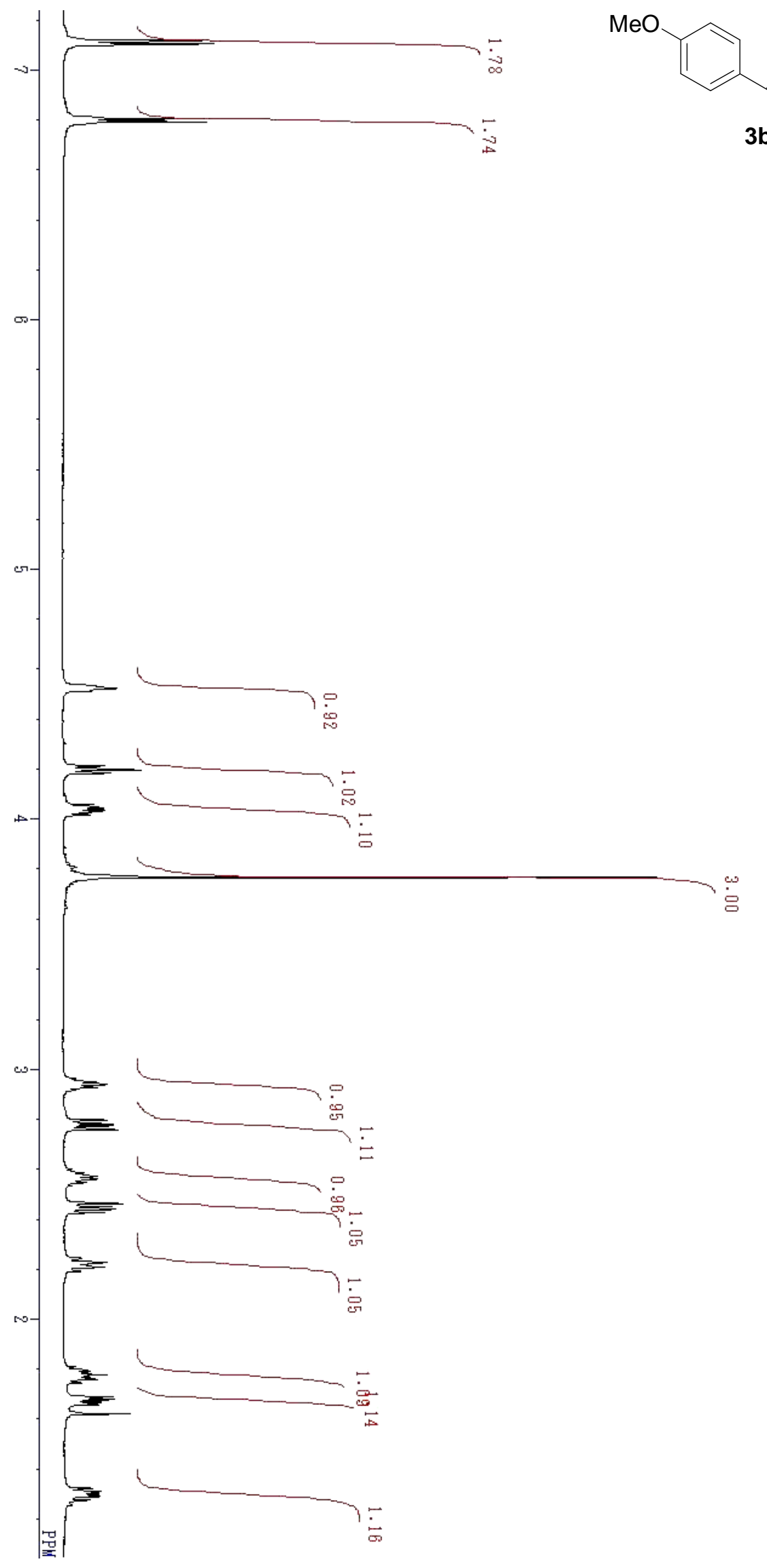




$$
E^{-}
$$




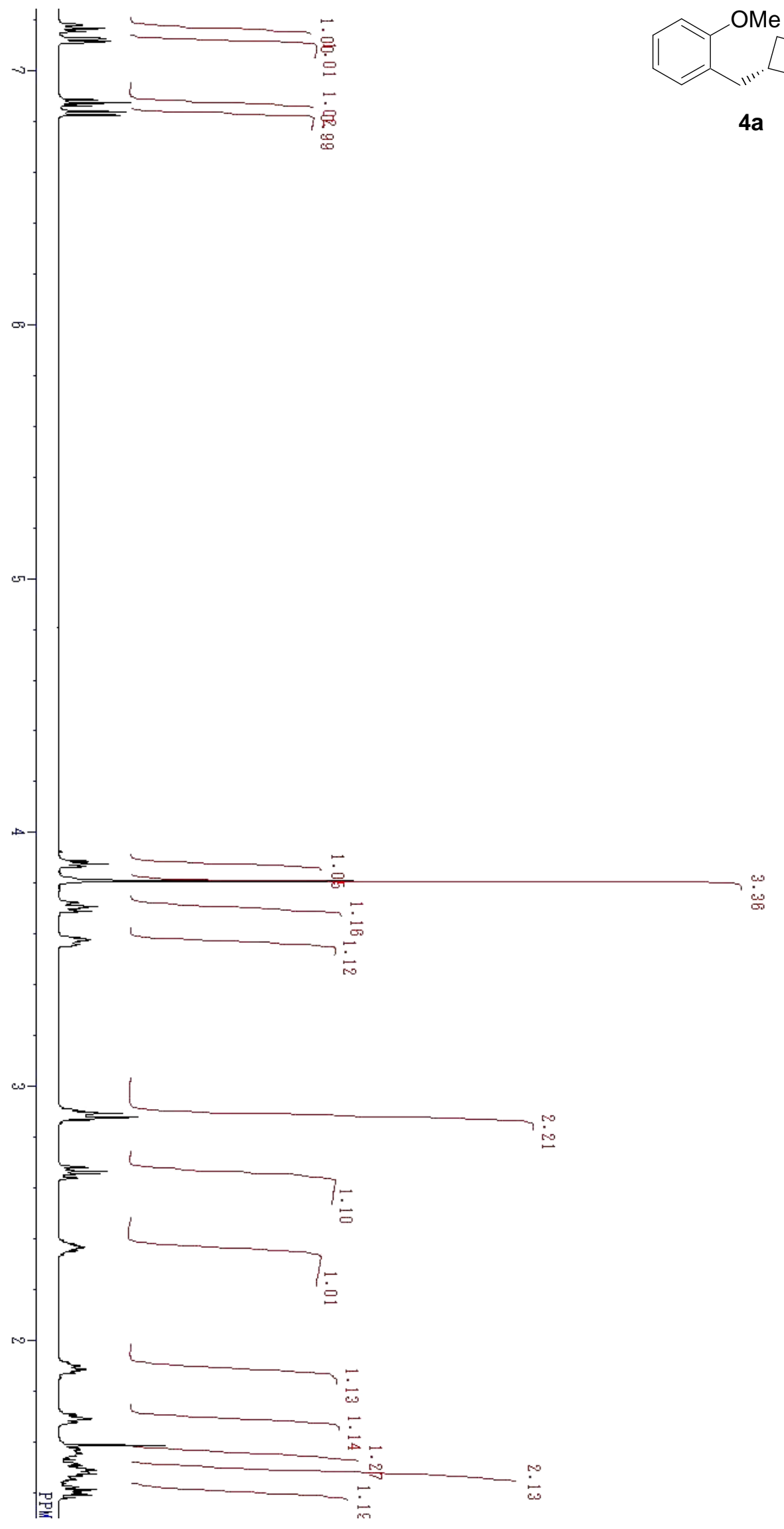




$$
E
$$




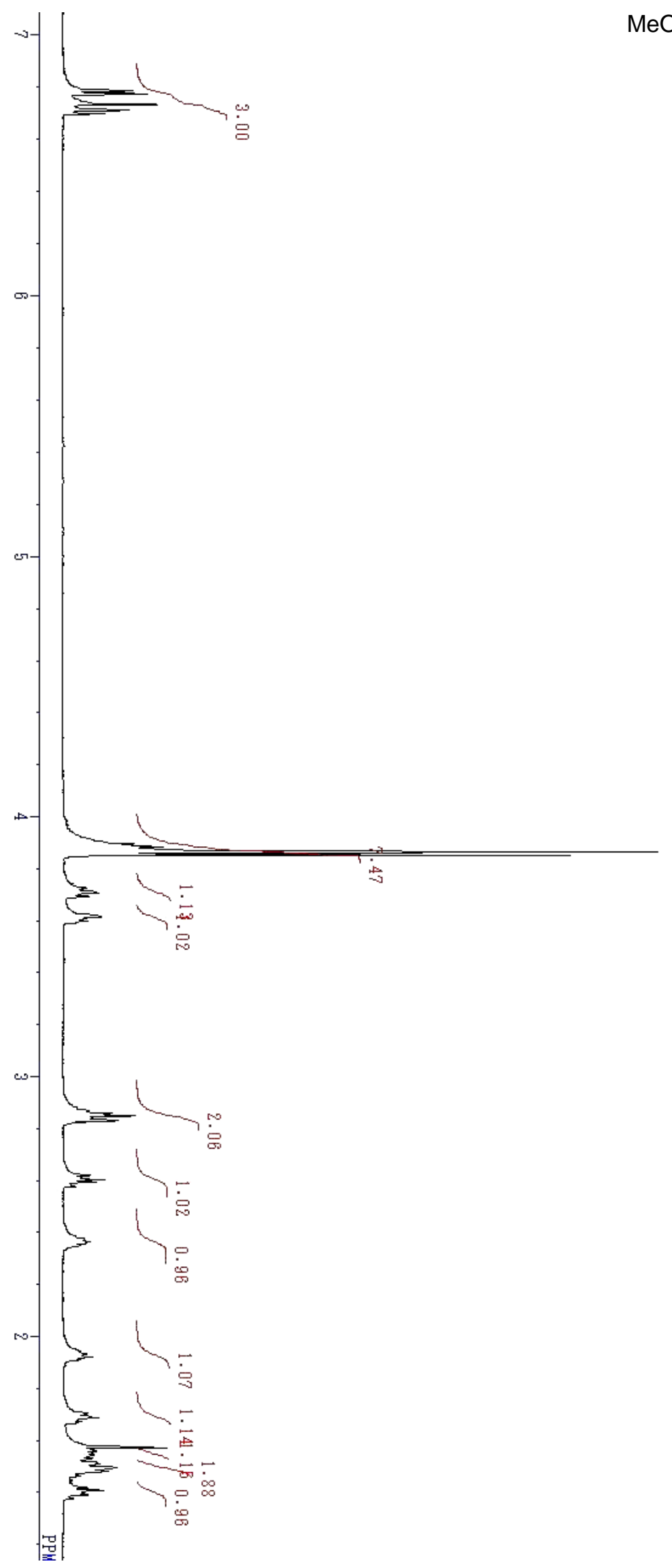

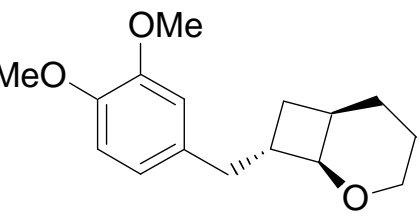

$5 a$ 


$$
k
$$

